\title{
A phenomenological model of the time course of maximal voluntary isometric contraction force for optimization of complex loading schemes
}

\author{
Johannes L. Herold ${ }^{1 *}$, Christian Kirches ${ }^{2}$, and Johannes P. Schlöder ${ }^{1}$ \\ ${ }^{1}$ Interdisciplinary Center for Scientific Computing, Heidelberg University, \\ Heidelberg, Germany. \\ ${ }^{2}$ Institute for Mathematical Optimization, Technische Universität \\ Carolo-Wilhelmina zu Braunschweig, Braunschweig, Germany.
}

(Submitted to bioRxiv.org on 2018/01/30. Last modified on 2018/01/30.)

\begin{abstract}
We construct a simple and predictive ordinary differential equation model to describe the time course of maximal voluntary isometric contraction (MVIC) force during voluntary isometric contractions and at rest. These time courses are of particular interest whenever force capacities are a limiting factor, e.g. during heavy manual work or resistance training (RT) sessions. Our model is able to describe MVIC force under complex loading schemes and is validated with a comprehensive set of published data from the elbow flexors. We use the calibrated model to analyze fatigue and recovery patterns observed in the literature. Due to the model's structure, it can be efficiently employed to optimize complex loading schemes. We demonstrate this by computing a work-rest schedule that minimizes fatigue and an optimal isometric RT session as examples.
\end{abstract}

Keywords: mathematical model, maximal voluntary isometric contraction force, muscle fatigue, optimal control, parameter estimation, recovery, resistance training

\section{Introduction}

Muscle fatigue is defined as an exercise-induced reduction in the ability to generate force or power [24]. Multiple task-specific mechanisms contribute to this complex phenomenon [19]. These mechanisms are categorized as peripheral (arising distal from the neuromuscular junction) or central (originating at spinal or supraspinal level). For a comprehensive overview of the physiological mechanisms, we refer to the work of Allen et al. [1] and Gandevia [24].

Several criteria can be used to measure muscle fatigue, e.g. the maximal voluntary isometric contraction (MVIC) force, power, the rate of force development, the one-repetition-maximum, or repetition strength [73, 57, 9]. To

\footnotetext{
${ }^{*}$ Corresponding author. Email: johannes.herold@iwr.uni-heidelberg.de.
} 
distinguish between peripheral and central contributions, one can superimpose external stimuli during MVICs either electrically to the nerve trunk or to the muscle belly [63], or magnetically to the motor cortex [72]. The resulting force increment then provides information about the origin of fatigue. During submaximal contractions and at rest, MVICs combined with external stimuli have to be interspersed to determine the corresponding time courses. For a review of these time courses, we refer to the work of Carroll et al. [13.

Predicting the time course of muscle fatigue and recovery would enable an optimal use of the limited human force capacities. With a suitable mathematical model, work shifts could be scheduled to avoid fatigue related accidents [29, 74, or resistance training (RT) sessions could be individualized to optimize adaptations 21. Owing to the large number of possible combinations of contraction intensities and durations, these optimization problems are inherently high-dimensional. Thus, derivative-based methods have to be employed for an efficient solution, which imposes several mathematical requirements on model candidates.

This work deals with modeling the time course of MVIC force for several reasons. First, MVIC force is considered one of the gold standards for determining muscle fatigue [73, as its measurements are easy to standardize and to conduct. It is therefore used extensively by physiologists and sports scientists, who could benefit from a predictive model when designing experiments. Second, although most everyday movements are of a dynamic nature, isometric contractions contribute to stabilization during those movements [2]. For this reason, isometric strength capacities and especially their connection to possible injuries are of high interest, e.g. in ergonomics [36, 28, or sports 41]. Third, isometric strength is an important physical characteristic for a variety of athletes, e.g. wrestlers, gymnasts 68, or climbers [21]. Due to the specificity of adaptations, instead of dynamic exercises, isometric training is favorable for these athletes. Finally, isometric resistance training can be used as part of a rehabilitation program, e.g. when joint movements are restricted or not advisable [38].

\subsection{Contributions}

In this article, we provide a literature overview of mathematical and computational models describing the time course of MVIC force. We demonstrate that a new model is necessary as existing models suitable for derivative-based optimization do not perform satisfactorily. Based on physiological observations and existing work by Freund and Takala 22, we propose a phenomenological ordinary differential equation (ODE) model of the time course of MVIC force during voluntary isometric contractions and at rest. We use a comprehensive set of published data to validate the model for the elbow flexors and the model shows promising results. Furthermore, we illustrate the benefits of the model structure by computing a work-rest schedule that minimizes fatigue and an optimal isometric RT session as examples. 


\subsection{Structure of the article}

The remainder of this article is structured as follows. In Section 2, we describe the data used for our work. In Section 3, we provide an overview of existing models and evaluate them with respect to our purposes. In Section 4 , we justify the need for a new model. In Section 5, we present our model and validate it by estimating parameters from a subset of the available data and using those estimates to predict the remaining data. In Section 6, we use the calibrated model to examine fatigue and recovery patterns observed in the literature. Furthermore, we compute a work-rest schedule that minimizes fatigue and plan an optimal isometric RT session. In Section 7, we conclude with an outlook on possible extensions and applications.

\section{Description of the data used}

To the best of our knowledge, there is no study that reports time courses of MVIC force for the same muscle group of a single subject under different loading schemes. Thus, we use the mean values from several experiments examining muscle fatigue and recovery of the elbow flexors to evaluate the performance of the models under consideration.

The following experiments are used:

E1 Taylor et al. 69 examine a 2 min MVIC and a recovery period lasting roughly $7 \mathrm{~min}$ for 8 subjects. Up to $30 \mathrm{MVIC}$ force measurements are given per subject.

E2 Søgaard et al. 66] examine a submaximal contraction at $15 \%$ of baseline MVIC force lasting $43 \mathrm{~min}$ and $23 \mathrm{~min}$ of recovery for 9 subjects. 29 MVIC force measurements are given per subject.

E3a Taylor et al. 70] examine intermittent MVICs of $5 \mathrm{sec}$ contraction and 5 sec rest and a 2 min recovery period for 9 subjects. Up to 18 MVIC force measurements are given per subject.

E3b Taylor et al. [70] examine intermittent MVICs of 15 sec contraction and $10 \mathrm{sec}$ rest and a $4 \mathrm{~min}$ recovery period for 9 subjects. Up to $30 \mathrm{MVIC}$ force measurements are given per subject.

E3c Taylor et al. 70 examine intermittent MVICs of $15 \mathrm{sec}$ contraction and 5 sec rest and a 4 min recovery period for 8 subjects. Up to 30 MVIC force measurements are given per subject.

E3d Taylor et al. 70] examine intermittent MVICs of $30 \mathrm{sec}$ contraction and 5 sec rest and a $3 \mathrm{~min}$ recovery period for 9 subjects. Up to 17 MVIC force measurements are given per subject.

E4 Gandevia et al. 25] examine a $2 \mathrm{~min}$ MVIC and a subsequent $3 \mathrm{~min}$ recovery period for 8 subjects. 13 mean MVIC force values and standard 
errors of the means were extracted from the figures with the software Engauge Digitizer 10.0 [50].

E5 Smith et al. 65 examine a 70 min submaximal contraction at $5 \%$ of baseline MVIC force and the following $29 \mathrm{~min}$ of recovery for 8 subjects. 52 MVIC force measurements are given per subject. As the measurement times are only given for one subject, we use those for the whole sample.

All experiments employed a similar setup (i.e. elbow flexed to 90 degrees, forearm vertical and supinated [69, 66, 70, 25, 65]), which justifies a comparison of these values. To the best of our knowledge, this is the most comprehensive set of data used to validate a model of the time course of MVIC force.

For each experiment $k \in\{\mathrm{E} 1, \mathrm{E} 2, \mathrm{E} 3 \mathrm{a}, \mathrm{E} 3 \mathrm{~b}, \mathrm{E} 3 \mathrm{c}, \mathrm{E} 3 \mathrm{~d}, \mathrm{E} 4, \mathrm{E} 5\}$, we are using the mean values

$$
\eta_{l}^{k}=\frac{1}{n^{k}} \sum_{i=1}^{n^{k}} \eta_{l, i}^{k}
$$

of the $n^{k}$ individual measurements $\eta_{l, i}^{k}$ to validate our model. The measurement errors $\varepsilon_{l}^{k}$ are assumed to be additive, independent, and identically normally distributed with mean zero and standard deviation $\sigma_{l}^{k}$. We take $\sigma_{l}^{k}$ to be the corrected sample standard deviation (SD) of the corresponding mean value $\eta_{l}^{k}$ divided by the square root of the sample size $n^{k}$, i.e.

$$
\sigma_{l}^{k}=\sqrt{\frac{1}{n^{k}\left(n^{k}-1\right)} \sum_{i=1}^{n^{k}}\left(\eta_{l, i}^{k}-\eta_{l}^{k}\right)^{2}} .
$$

Thus, $\sigma_{k}^{l}$ yields an approximation of the standard error of the mean. For experiment E4, the means and the corresponding standard errors were extracted directly from the figures. The test contractions interspersed during submaximal contractions and recovery are described to last $1-2$ [70] or $2-3 \sec 66$ ]. Therefore, we model those contractions to last 2 sec.

Remark. Since we are working with isometric contractions, force and torque normalized to baseline are equivalent. Thus, we do not differentiate between the two terms in the following.

\section{Literature overview}

Several mathematical and computational models have been proposed to predict the time course of MVIC force during voluntary isometric contractions or the time course of maximal evocable isometric force under external stimulation. As fatigue is highly task-dependent and substantial differences exist between evoked and voluntary contractions [48], we do not include models created for external stimulation in the following overview. Furthermore, we assume that all models can be applied at muscle group level and account for peripheral and central factors contributing to MVIC force generation. 


\subsection{Evaluation criteria}

We put a special focus on the following criteria necessary for our intentions.

C1 Can the model predict fatigue of MVIC force under complex patterns of voluntary isometric contractions, i.e. maximal, submaximal, sustained, and intermittent contractions of varying intensity?

C2 Does the model include recovery of MVIC force?

C3 Is the model suitable for real-life applications, i.e. is the number of parameters low and are all parameters identifiable through MVIC force measurements?

C4 Is the model suitable for high-dimensional optimization, i.e. is the model suitable for derivative-based solution methods?

Many physiology-based models implement a feedback loop with some kind of proportional-integral-derivative controller to imitate force adjustments by the nervous system and match simulated to target force. Unfortunately, these closed-loop controllers violate criterion $\mathrm{C} 4$ as they are usually not suitable for derivative-based optimization methods without considerable effort spent on reformulations.

\subsection{Existing models}

First attempts to quantify the development of muscle fatigue were made around 1960 by Monod and Scherrer [51, 52] and Rohmert [58, who mathematically described the nonlinear relationship between contraction intensity and endurance time with algebraic equations. Subsequently, several authors proposed similar equations or developed joint-specific versions [18, 40. However, these models neither allow to evaluate the time course of MVIC force for more complex contraction scenarios nor do they include recovery.

Fuglevand et al. 23] constructed a model of recruitment and rate coding of a pool of motor units. As the model does not include fatigue effects, it was later on extended by Dideriksen et al. [17]. The fatigue-induced changes of the model require a controller to maintain target force. Potvin and Fuglevand [53] proposed another modification to the original model [23, which has yet to be extended to be able to describe force recovery.

Hawkins and Hull [30] incorporated fatigue effects into a previously developed fiber based model [31. The model does not describe recovery after work and employs an if-else structure to account for the recruitment order of different fiber types.

Based on the work of Rich [55] and Deeb et al. [16], Wood et al. [74] proposed a model and subsequently used it to minimize fatigue during work schedules consisting of intermittent contractions with constant intensities and duty cycles. The least fatiguing setting was found by an exhaustive search through the parameter space. Although their approach pursued a similar goal as this work, the fixed duty cycles impose an undesired limitation on the loading schemes. 
Freund and Takala [22] constructed a biomechanical model of the forearm and introduced a simple ODE to account for effects of fatigue and recovery. Ma et al. [46, 47] utilized similar dynamics but separated fatigue and recovery, which resulted in a branchwise definition of the ODE's right-hand-side. Riener et al. [56] modeled the effects of fatigue and recovery for externally stimulated contractions. Although they chose muscle activation as model input, the proposed dynamics are closely related to the ones of Freund and Takala [22] and Ma et al. 47, which is why we mention it here. Fayazi et al. 20] based their model on that of Ma et al. [47, but modeled fatigue and recovery to occur simultaneously as originally proposed by Freund and Takala [22] for voluntary contractions and by Riener et al. [56 for stimulated contractions. Fayazi et al. 20. used their model subsequently to calculate optimal pacing strategies for a cyclist. The type of model proposed by these authors fulfills our requirements and is thus examined further in the next section.

Liu et al. 44] introduced a three-compartment model distinguishing between active, fatigued, and resting motor-units. Fatigue and recovery effects are represented by flows between the compartments and brain effort was chosen as model input. Since brain effort is only known for maximal efforts, the model was later extended by Xia and Law [75] by including a controller to account for the recruitment hierarchy of three different fiber types when matching the target force. A different modification of the original model 44 was implemented by Sih et al. 64. The authors expanded the model to four compartments and reformulated their equations to circumvent brain effort as input. In general, this allows the model to simulate arbitrary force profiles. Nevertheless, it is still defined branchwise.

Several authors have extended or modified the model of Xia and Law 75]. Gede and Hubbard [27] added a force-velocity dependence and generalized the model to task level. Furthermore, they removed the fast components of the model and changed the input to be the amount of active muscle which makes the model suitable for optimization [26. However, because of the separate inputs for each fiber type, the model could only be validated for maximal efforts. Other modifications to the original model [75], e.g. using time-variant 67] or contraction-specific [45] parameters, have been developed as well.

James and Green [34] used a similar approach as Sih et al. 64] but assumed that contractile properties vary as continuous functions of time and motor unit type. The power output of a single motor unit is defined non-smooth and the model does not account for force recovery. Callahan et al. [10, 11] built a comprehensive model of torque generation. The model can be used to describe voluntary and externally stimulated contractions and uses a controller to match generated to target torque. Contessa and Luca [15] developed a model focusing on motor units containing a feedback loop implemented to regulate the excitation and match target force. 


\section{Evaluation of suitable models}

As discussed in the previous section, the models proposed by Freund and Takala 22] and Fayazi et al. 20] are suitable for our intentions. Thus, we present these models in detail and evaluate their performance by fitting them to the available data.

\subsection{Freund and Takala model}

Freund and Takala 22] modeled fatigue and recovery of MVIC force as

$$
\frac{\mathrm{d}}{\mathrm{d} t} x_{\mathrm{MVIC}}(t)=p_{1}\left(1-x_{\mathrm{MVIC}}(t)\right)-p_{2} u_{\mathrm{abs}}(t) .
$$

Here,

$$
x_{\mathrm{MVIC}}: T \rightarrow[0,1]
$$

represents the current MVIC force capacity on the time interval $T \subset[0, \infty)$ and

$$
u_{\mathrm{abs}}: T \rightarrow[0,1]
$$

represents the absolute external isometric load and is the model input. The fatigue rate $p_{2} u_{\text {abs }}(t)$ depends linearly on $u_{\text {abs }}$. The recovery rate $p_{1}\left(1-x_{\mathrm{MVIC}}(t)\right)$ depends linearly on the difference between the current force capacity $x_{\text {MVIC }}$ and the maximal force capacity. Since we scale all states and inputs to $[0,1]$, the maximal force capacity equals 1 . The dimensionless parameters $p_{1} \in[0, \infty)$ and $p_{2} \in[0, \infty)$ determine the maximal speed of exponential recovery and fatigability of the muscle group. The initial condition

$$
x_{\mathrm{MVIC}}(0)=x_{0} \in[0,1]
$$

describes the force capacity of the muscle group at $t=0$. For an unfatigued muscle, we choose $x_{0}=1$.

\subsection{Fayazi et al. model}

Based on the work of Ma et al. [47], Fayazi et al. [20] proposed dynamics similar to those of Freund and Takala [22]. Keeping the notation introduced earlier, the dynamics of the model read as

$$
\frac{\mathrm{d}}{\mathrm{d} t} x_{\mathrm{MVIC}}(t)=p_{1}\left(1-x_{\mathrm{MVIC}}(t)\right)-p_{2} u_{\mathrm{abs}}(t) x_{\mathrm{MVIC}}(t) .
$$

Thus, the difference to the model of Freund and Takala [22] is an additional dependency of the fatigue rate on the current MVIC force. In the model of Ma et al. [47, where fatigue and recovery are modeled separately, this factor ensures a sufficient decrease of fatigability during an MVIC. 


\subsection{Simulating MVICs}

To simulate maximal voluntary contractions, it is favorable to substitute

$$
u_{\text {abs }}(t)=u_{\text {rel }}(t) x_{\mathrm{MVIC}}(t)
$$

and use

$$
u_{\text {rel }}: T \rightarrow[0,1]
$$

the load relative to the current force capacity, as input. This substitution reflects the experimental settings of an MVIC, during which subjects are asked to contract maximally $\left(u_{\mathrm{rel}}=1\right)$ instead of maintaining a certain target force $u_{\text {abs }}$. It furthermore allows us to simulate MVICs without an unnecessarily complex mathematical description of the non-constant input force $u_{\text {abs }}$ and is necessary to model MVICs for which no measurement values are given (e.g. in experiment E3a).

However, in contrast to using $u_{\text {abs }}$ as input, this substitution only gives a prediction of the absolute external load at a certain level of effort $u_{\text {rel }}$ by the model and not the actual absolute external load. Depending on whether the contraction is submaximal or maximal, we either use $u_{\text {abs }}$ or $u_{\text {rel }}$ to describe the experimental settings in the following.

\subsection{Necessity of a new model}

We now fit these models simultaneously to the data of E1 and E2 presented in Section 2. The multiple experiment parameter estimation problem for one model is formulated as

$$
\begin{aligned}
& \min _{p, x^{k}(\cdot)} \sum_{k \in\{\mathrm{E} 1, \mathrm{E} 2\}} \sum_{l=1}^{M^{k}}\left(\frac{x_{\mathrm{MVIC}}^{k}\left(t_{l}^{k}\right)-\eta_{l}^{k}}{\sigma_{l}^{k}}\right)^{2} \\
& \text { s.t. for } k \in\{\mathrm{E} 1, \mathrm{E} 2\} x^{k}: T^{k} \rightarrow[0,1]
\end{aligned}
$$

satisfies the model equations (3) or respectively (4)

$$
\text { and the initial condition } x_{\mathrm{MVIC}}^{k}(0)=1 \text {. }
$$

Here, $M^{k} \in \mathbb{N}$ is the number of measurements in experiment $k \in\{\mathrm{E} 1, \mathrm{E} 2\}$ and the $l$-th measurement $\eta_{l}^{k}$ of experiment $k$ is characterized by its time $t_{l}^{k}$ and the standard deviation $\sigma_{l}^{k}$ of its measurement error. As explained previously, the loading scheme of experiment $k$ is described by the input function $u_{\text {abs }}$ or via substitution by $u_{\text {rel }}$. The state $x_{\text {MVIC }}^{k}$ then denotes the model response for this setting.

The optimization problem constrained by an ODE (6) is solved by employing a first-discretize-then-optimize strategy. We use a direct multiple shooting approach to reduce the problem to a finite-dimensional form [7] and employ DAESOL [4 for integration of the ODE and sensitivity generation via internal numerical differentiation [6. Afterwards, the resulting structured nonlinear least squares problem is solved with PAREMERA [37, an implementation of the 
reduced generalized Gauss-Newton method 62. Both packages are embedded in the optimal experimental design software VPLAN [39].

Figure 1 illustrates the results of the fit for the model of Freund and Takala 22 and Figure 2 for the model of Fayazi et al. 20. Table 1 lists the parameter estimates and their estimated relative standard deviations for both models. Table 2 lists the mean absolute errors and weighted residual sum of squares of the fits for both models.

When evaluating the fits, the necessity of a new model becomes clear. The model of Freund and Takala 22] captures the fatigue development during the MVIC of E1 correctly, whereas the model of Fayazi et al. 20] underestimates it slightly. This is caused by combining the fatigue and recovery branches of Ma et al. 47. linearly. Both models overestimate fatigue in the beginning of the submaximal contraction E2 and reach a steady-state too early. Furthermore, due to their mono-exponential recovery terms, they cannot capture the initially faster recovery after cessation of the contractions.
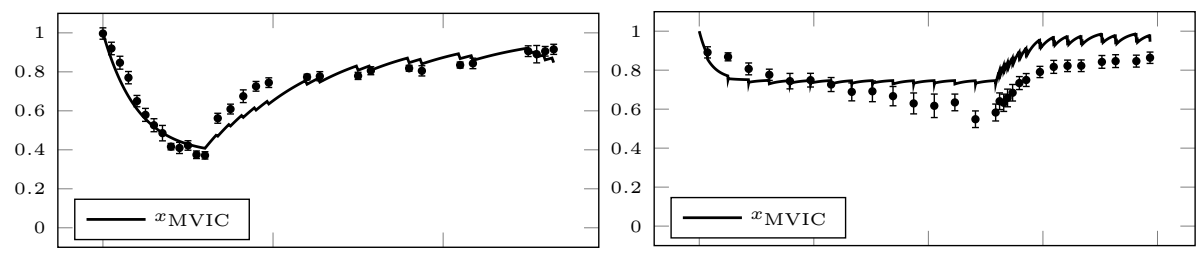

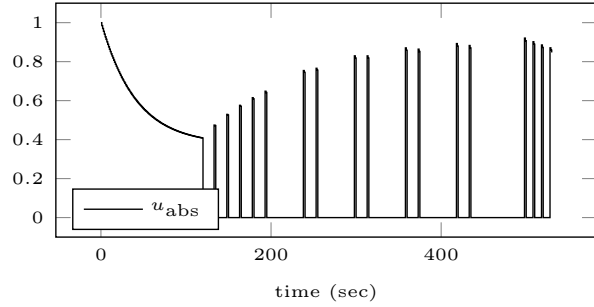

(a)

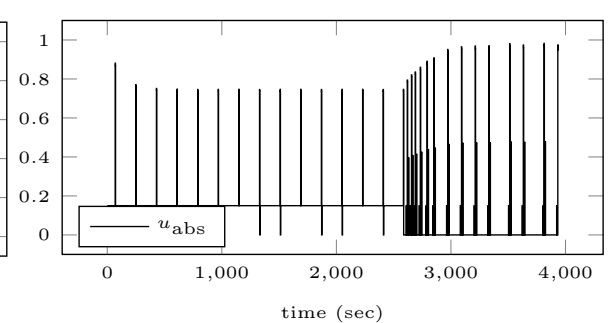

(b)

Figure 1: Model response obtained by fitting the model of Freund and Takala 22] simultaneously to the data of E1 (a) and E2 (b). The top row shows the mean values of the experiments plotted against the model response for the experimental setting. The error bars represent the standard errors of the means. Additionally, the absolute force input as predicted by the model (explained in Section 4.3 is illustrated in the bottom row.

\section{Proposed model}

In this section, we propose a new ODE model of the time course of MVIC force, motivate our choice, and validate the model. 

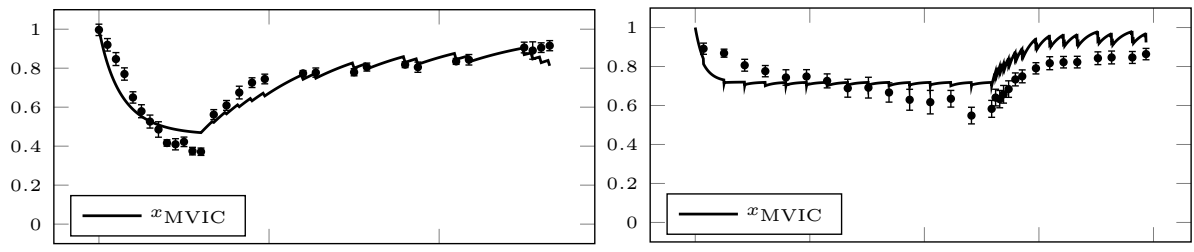

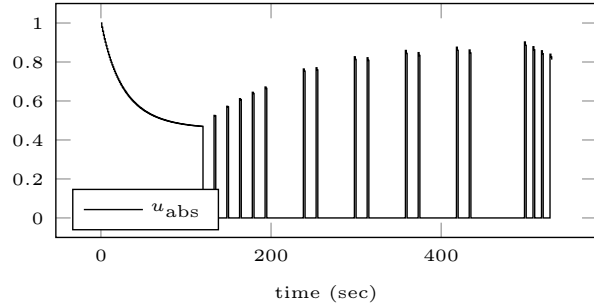

(a)

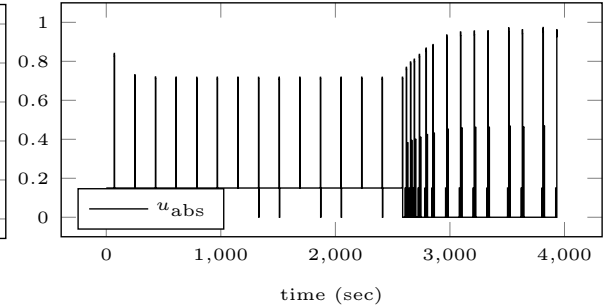

(b)

Figure 2: Model response obtained by fitting the model of Fayazi et al. [20] simultaneously to the data of E1 (a) and E2 (b). The top row shows the mean values of the experiments plotted against the model response for the experimental setting. The error bars represent the standard errors of the means. Additionally, the absolute force input as predicted by the model (explained in Section 4.3 is illustrated in the bottom row.

Table 1: Parameter estimates and their estimated relative standard deviations obtained by simultaneously fitting the models of Freund and Takala [22] and Fayazi et al. 20, to the data of E1 and E2.

\begin{tabular}{lllll}
\hline & $p_{1}$ & $\pm \mathrm{SD}$ & $p_{2}$ & $\pm \mathrm{SD}$ \\
\hline Freund and Takala [22] & $9.20 \cdot 10^{-3}$ & $\pm 3.75 \%$ & $1.53 \cdot 10^{-2}$ & $\pm 3.56 \%$ \\
Fayazi et al. [20] & $8.66 \cdot 10^{-3}$ & $\pm 4.90 \%$ & $2.24 \cdot 10^{-2}$ & $\pm 4.98 \%$ \\
\hline
\end{tabular}

Table 2: Mean absolute errors (MAE) and weighted residual sum of squares (WRSS) obtained by simultaneously fitting the models of Freund and Takala [22] and Fayazi et al. 20] to the data of E1 and E2.

\begin{tabular}{llllll}
\hline & \multicolumn{2}{c}{ E1 } & & \multicolumn{2}{c}{ E2 } \\
\cline { 2 - 3 } \cline { 5 - 6 } & MAE & WRSS & & MAE & WRSS \\
\hline Freund and Takala [22] & 0.04 & 151.07 & & 0.10 & 273.72 \\
Fayazi et al. [20] & 0.05 & 210.97 & & 0.09 & 234.80 \\
\hline
\end{tabular}




\subsection{Structure of the model}

As the model of Freund and Takala 22] performed slightly better in the previous fits, we use their model as a foundation. The proposed new model is given as

$$
\begin{aligned}
\frac{\mathrm{d}}{\mathrm{d} t} x_{\text {slow }}(t) & =p_{1}\left(1-x_{\text {slow }}(t)\right)-p_{2} u_{\text {abs }}(t) \\
\frac{\mathrm{d}}{\mathrm{d} t} x_{\text {fast }}(t) & =p_{3}\left(1-u_{\text {abs }}(t)\right)^{p_{4}}\left(1-x_{\text {fast }}(t)\right)-p_{5} u_{\text {abs }}(t) \\
h_{\text {MVIC }}(t) & =x_{\text {slow }}(t) x_{\text {fast }}(t)
\end{aligned}
$$

where

$$
x: T \rightarrow[0,1]^{2}
$$

is defined on the time horizon $T \subset[0, \infty)$ and consists of two state variables $x_{\text {fast }}$ and $x_{\text {slow }}$. Again, we scale all states and inputs to $[0,1]$. The model furthermore contains five dimensionless parameters $p_{j} \in[0, \infty)$ for $j \in\{1, \ldots, 5\}$ and one input function

$$
u_{\mathrm{abs}}: T \rightarrow[0,1]
$$

representing the absolute external isometric load. The current MVIC force capacity is denoted by

$$
h_{\mathrm{MVIC}}: T \rightarrow[0,1]
$$

and the initial conditions for the states are given by

$$
x(0)=x_{0} \in[0,1]^{2} .
$$

For an unfatigued muscle, one chooses $x_{0}=(1,1)^{T}$.

Table 3 summarizes the model components. To increase readability throughout the rest of this paper, we omit arguments of the states and input functions whenever the dependencies are clear.

\subsection{Motivation for choosing this structure}

We duplicated the dynamics of Freund and Takala 22] and modeled MVIC force as the product of two state variables. This was motivated by the observations of fast and slow dynamics of fatigue and recovery [13] and furthermore supported by other authors who proposed using double-exponential functions to describe the time courses of fatigue [16] and recovery [14. The product was chosen to represent the chain of mechanisms leading to force generation. Yet, since this chain includes complex feedforward and feedback mechanisms, the strict separation is obviously an oversimplification.

The processes summarized in $x_{\text {slow }}$ play a bigger role in contractions of long duration or repeated intermittent ones, as this state fatigues much slower and takes longer to recover. Correlations to the $\mathrm{Ca}^{2+}$ sensitivity of the crossbrigdes, 
Table 3: Overview of the model components.

\begin{tabular}{lll}
\hline & Type & Interpretation \\
\hline$x_{\text {slow }}$ & State variable & Slow component \\
$x_{\text {fast }}$ & State variable & Fast component \\
$h_{\text {MVIC }}$ & Function & MVIC force \\
$p_{1}$ & Parameter & Maximal recovery rate of $x_{\text {slow }}$ \\
$p_{2}$ & Parameter & Maximal fatigue rate of $x_{\text {slow }}$ \\
$p_{3}$ & Parameter & Maximal recovery rate of $x_{\text {fast }}$ \\
$p_{4}$ & Parameter & Influence of $u_{\text {abs } \text { on recovery rate of } x_{\text {fast }}}$ \\
$p_{5}$ & Parameter & Maximal fatigue rate of $x_{\text {fast }}$ \\
$u_{\text {abs }}$ & Input function & Absolute external load \\
\hline
\end{tabular}

to the $\mathrm{Ca}^{2+}$ release of the sarcoplasmic reticulum [1, and to glycogen depletion [61] are likely. Furthermore, there is also a long lasting effect of reduced voluntary activation after prolonged contractions, the reason for which is currently unknown 13 .

$x_{\text {fast }}$ pools a variety of mechanisms responsible for a quick onset of MVIC force reduction and a fast recovery. These mechanisms contribute to a larger degree during maximal and near maximal contractions and seem to be closely related to muscle perfusion [13. Possible factors include metabolite accumulation (e.g. inorganic phosphate) [1, firing of group III and IV afferents [71, or energy deficiency [61]. Since studies have established a link between blood occlusion in the muscle (either through high-intensity contractions or artificially) and impeded recovery [5, 35], we added a dependency of the recovery rate on $\left(1-u_{\text {abs }}\right)^{p_{4}}$. The exponent $p_{4}$ determines the contraction intensity at which total occlusion occurs $[3,60$. A similar dependency on activation was proposed by Riener et al. [56] in the context of external stimulation. However, they assumed a linear relationship.

\subsection{Fitting the model to the data}

To validate our model, we fit it to the data of E1, E2, E3a, and E3c. This subset was chosen as it contains a sustained maximal, a sustained submaximal, and two intermittent maximal contractions and enables an identification of the parameters. For details on the multiple experiment parameter estimation problem, we refer to Section 4.4. Figure 3 depicts the results of the fit. Table 4 lists the parameter estimates and their estimated relative standard deviations. Table 5 lists the mean absolute errors and weighted residual sum of squares of the fits.

Although more data was used, the fit is improved substantially compared to Figures 1 and 2. This is to be expected, as the proposed model employs five instead of two parameters. Yet, since all parameters could be estimated reasonably well, the model is not overparameterized. Our model is able to capture submaximal contractions, the initial fast recovery after cessation of a 

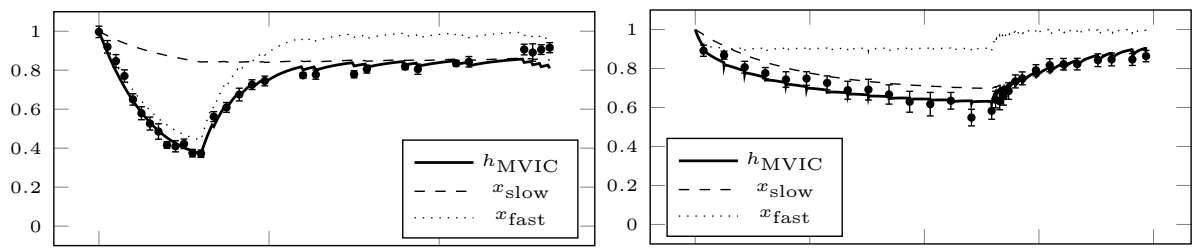

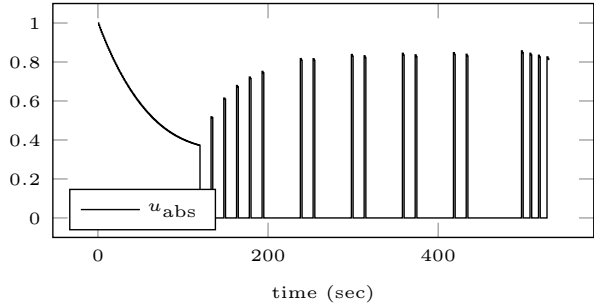

(a)
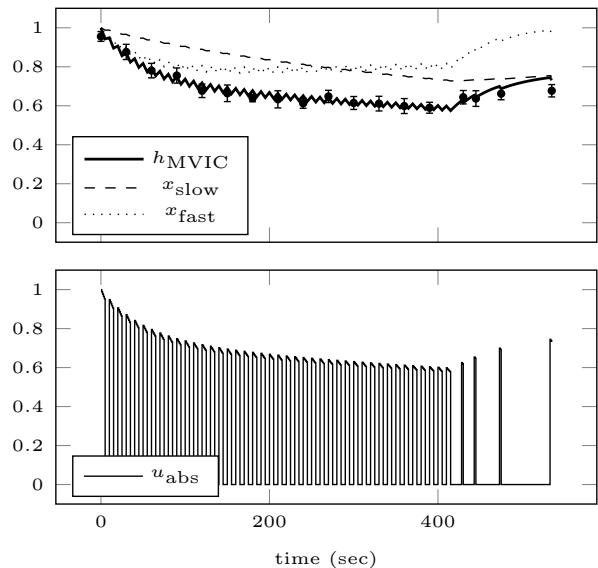

(c)

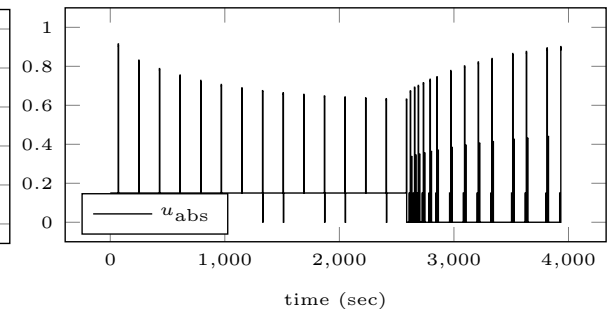

(b)
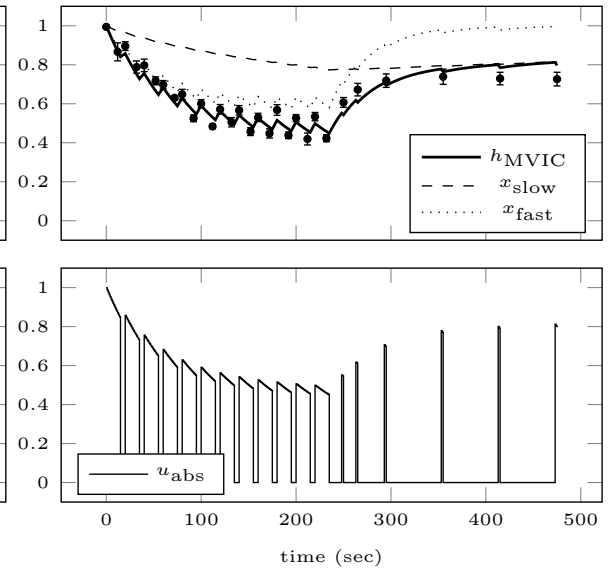

(d)

Figure 3: Model response obtained by fitting the proposed model simultaneously to the data of E1 (a), E2 (b), E3a (c), and E3c (d). The top row shows the mean values of the experiments plotted against the model response for the experimental setting. The error bars represent the standard errors of the means. Additionally, the absolute force input as predicted by the model (explained in Section 4.3 is illustrated in the bottom row.

contraction, and the delayed recovery after prolonged contractions.

Due to the phenomenological nature of the model, most of the estimated parameter values do not allow a direct physiological interpretation. Nevertheless, we expect physiological characteristics like fiber type composition, capillarization, buffering capacity, muscle mass and strength, energy stores, and others to be reflected in the dimensionless parameter estimates. An inter-individual 
Table 4: Parameter estimates and their estimated relative standard deviations obtained by simultaneously fitting the proposed model to the data of E1, E2, E3a, and E3c.

\begin{tabular}{llll}
\hline & Interpretation & Estimate & $\pm \mathrm{SD}$ \\
\hline$p_{1}$ & Maximal recovery rate of $x_{\text {slow }}$ & $1.20 \cdot 10^{-3}$ & $\pm 7.23 \%$ \\
$p_{2}$ & Maximal fatigue rate of $x_{\text {slow }}$ & $2.46 \cdot 10^{-3}$ & $\pm 6.04 \%$ \\
$p_{3}$ & Maximal recovery rate of $x_{\text {fast }}$ & $2.85 \cdot 10^{-2}$ & $\pm 6.44 \%$ \\
$p_{4}$ & Influence of $u_{\text {abs on recovery rate of } x_{\text {fast }}}$ & 3.99 & $\pm 11.84 \%$ \\
$p_{5}$ & Maximal fatigue rate of $x_{\text {fast }}$ & $9.44 \cdot 10^{-3}$ & $\pm 2.99 \%$ \\
\hline
\end{tabular}

Table 5: Mean absolute errors (MAE) and weighted residual sum of squares (WRSS) obtained by simultaneously fitting the proposed model to the data of $\mathrm{E} 1, \mathrm{E} 2, \mathrm{E} 3 \mathrm{a}$, and E3c.

\begin{tabular}{lllll}
\hline & E1 & E2 & E3a & E3c \\
\hline MAE & 0.03 & 0.03 & 0.02 & 0.03 \\
WRSS & 66.68 & 25.82 & 17.17 & 80.83 \\
\hline
\end{tabular}

comparison of the estimates from the same muscle group or an intra-individual comparison of those from different muscle groups might provide interesting physiological insights. However, to evaluate this hypothesis, more data is needed.

Since the term $\left(1-u_{\text {abs }}\right)^{p_{4}}$ was specifically introduced to mimic blood occlusion during intense contractions, we compare the estimate of $p_{4}$ to the values given in literature. Sadamoto et al. 60. determined that at a contraction intensity of $53 \%$ of baseline MVIC force, muscle blood flow in the biceps was arrested. For the external load $u_{\text {abs }}=0.53$, the estimated exponent $p_{4}=3.99$ would imply roughly $5 \%$ of the induced blood flow reaching the elbow flexors and providing some kind of recovery. As we are not only considering the biceps but all elbow flexors, and as Barnes [3] found a strength-dependency of the intensity necessary for total occlusion, this value seems to be consistent with the literature.

\subsection{Testing the predictive ability of the model}

The data of the remaining four experiments E3b, E3d, E4, and E5 is now used to test the predictive ability of the calibrated model. Figure 4 illustrates the prediction of the calibrated model for these experiments. Table 6 lists the mean absolute errors and weighted residual sum of squares of the predictions.

Using the estimated parameters, the model is able to predict the data of E3d, E4, and E5 satisfactorily. Nevertheless, it shows unexpected deficiencies when simulating experiment E3b. Namely, it cannot capture the increasing fatigability of the subjects during the experiment. The exact reasons are unknown. However, this increasing fatigability cannot be observed during the other experiments of Taylor et al. [70]. Furthermore, this increase actually contradicts the 

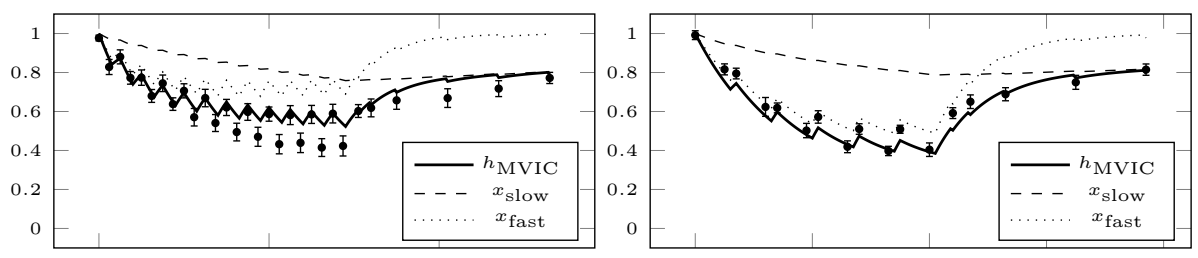

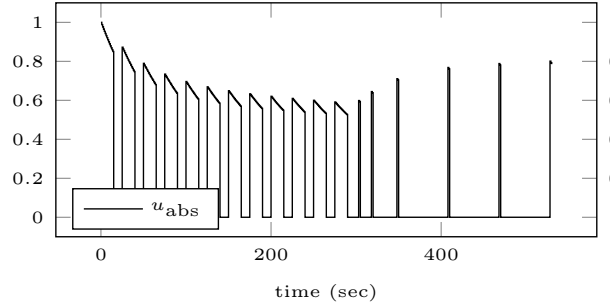

(a)
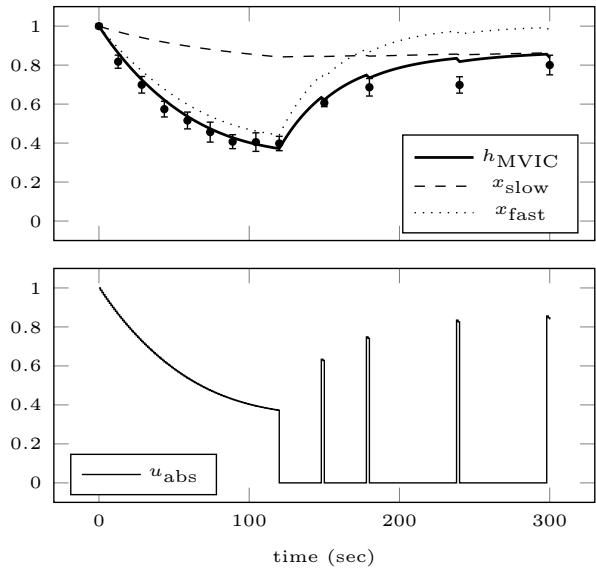

(c)

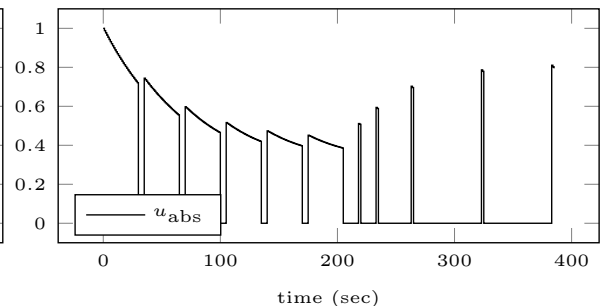

(b)
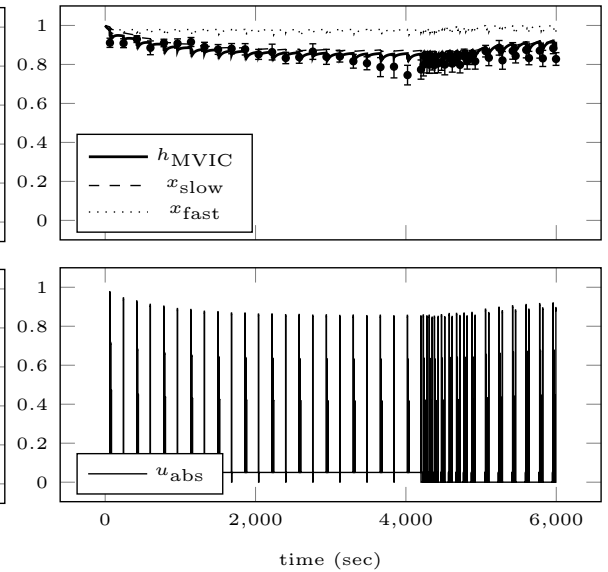

(d)

Figure 4: Model response obtained by simulating the calibrated model for the experimental settings of E3b (a), E3d (b), E4 (c), and E5 (d). The top row shows the mean values of the experiments plotted against the model response for the experimental setting. The error bars represent the standard errors of the means. Additionally, the absolute force input as predicted by the model (explained in Section 4.3) is illustrated in the bottom row.

size principle 32] according to which primarily fatigue-resistant fibers should be used as the protocol progresses. Thus, fatigability should decrease during each contraction and a steady-state should be reached, as can be seen in the other experiments. Since this experiment additionally shows the largest inter-subject variability compared to other experiments from the study by Taylor et al. [70], we suppose that motivational issues of some subjects are the cause of this phe- 
Table 6: Mean absolute errors (MAE) and weighted residual sum of squares (WRSS) obtained by using the calibrated model to predict the data of E3b, E3d, E4, and E5.

\begin{tabular}{lllll}
\hline & E3b & E3d & E4 & E5 \\
\hline MAE & 0.04 & 0.03 & 0.03 & 0.03 \\
WRSS & 47.53 & 37.86 & 15.28 & 41.00 \\
\hline
\end{tabular}

nomenon. We argue similarly concerning the force drops during the submaximal contractions E2 and E5 shortly before the end of the contraction, which are not captured by the model.

\section{$6 \quad$ Numerical case studies}

In this section, we illustrate how the calibrated model can be used for analysis and optimization of real-world scenarios. Throughout this section, we use the parameter values of the elbow flexors obtained in Section 5.3

\subsection{Model-based analysis of fatigue and recovery patterns}

We now examine two observations from the literature regarding the fatigue and recovery patterns of MVIC force. Since the model has been validated with contraction intensities of $15 \%$ of baseline MVIC force and MVICs, we use those for our simulations. Furthermore, the plots in Section 5 illustrate that short test MVICs interspersed during submaximal contractions and recovery do not substantially influence the time course of MVIC force and can therefore be neglected in our simulations.

First, Rozand et al. [59] observe that after sustained isometric contractions of the knee extensors with different intensities and similar force-time-integral (FTI) the induced level of fatigue did not differ. The FTI on the time interval $[0, T]$ is defined as

$$
\mathrm{FTI} \stackrel{\text { def }}{=} \int_{0}^{T} u_{\text {abs }}(t) \mathrm{d} t
$$

and often used as an analogue for work during isometric contractions, where no actual physical work is performed. Figure 5 shows the calibrated model simulated for a 60 sec MVIC and a submaximal contraction at $15 \%$ of baseline MVIC force lasting $292.40 \mathrm{sec}$ with the same FTI of 43.86. Both contractions end before a steady-state is reached. As can be seen in the plots, the induced fatigue is higher for the MVIC than for the submaximal contraction. After the maximal contraction, $h_{\mathrm{MVIC}}$ is reduced to $53.21 \%$. In contrast to this, $h_{\mathrm{MVIC}}$ is reduced to $82.37 \%$ after the submaximal contraction. We therefore cannot reproduce the results of Rozand et al. [59] for our settings.

Second, Rashedi and Nussbaum [54] observe that recovery is fatigue- and not task-dependent for intermittent isometric contractions of the index finger. 

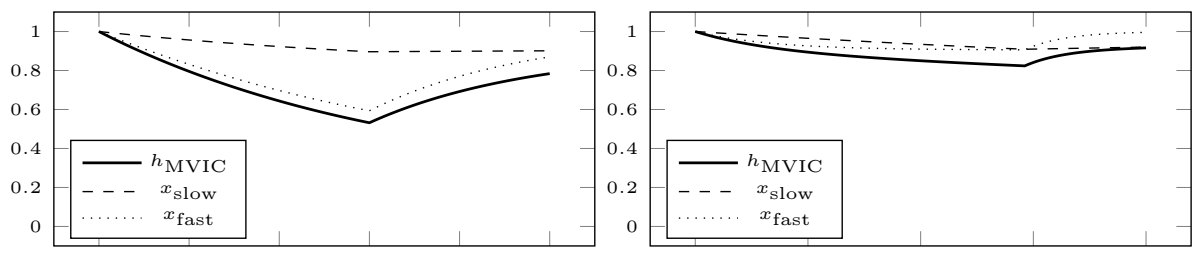

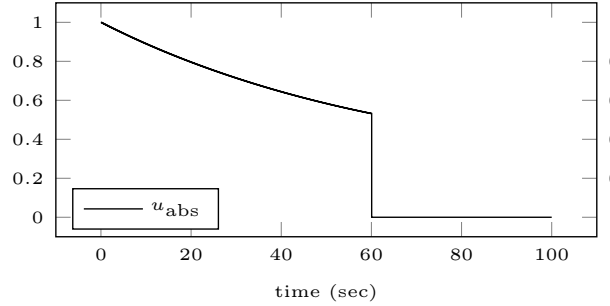

(a)

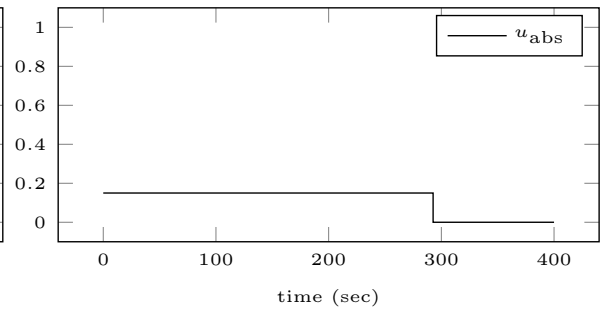

(b)

Figure 5: Model response obtained by simulating the calibrated model for an MVIC (a) and for a submaximal contraction (b) with the same force-timeintegral. The top row shows the model response and the absolute force input is illustrated in the bottom row. The induced fatigue is higher for the MVIC than for the submaximal contraction.

Iguchi et al. 33. notice similar dependencies after sustained isometric contractions of the quadriceps. Figure 6 shows the calibrated model simulated for a 40 sec MVIC and a submaximal contraction at $15 \%$ of baseline MVIC force lasting $2310 \mathrm{sec}$ both reducing MVIC force to $64.41 \%$ of baseline. Both contractions end before a steady-state is reached and are followed by a $10 \mathrm{~min}$ recovery period. As can be seen in the plots, recovery from the MVIC is almost complete after $10 \mathrm{~min}$, whereas recovery from the submaximal contraction takes much longer. At the end of the MVIC scenario, $h_{\mathrm{MVIC}}$ recovered to $96.25 \%$. In contrast to this, $h_{\mathrm{MVIC}}$ recovered to $85.97 \%$ at the end of the submaximal scenario. Thus, we cannot reproduce the results of Rashedi and Nussbaum [54] for our settings.

\subsection{Computing a work-rest schedule that minimizes fatigue}

To demonstrate the benefits of our model, which is suitable for derivative-based optimization, we adopt the example of Wood et al. 74], who compute a workrest schedule that minimizes fatigue during a grip task.

Wood et al. [74] define fatigue as the decay in maximal grip force at the end of the task. To keep their example simple, the physiological workload, the force, and the temporal pattern of the 60 sec work-rest cycles were held constant. Furthermore, the contraction intensity was bounded between $0.16 \leq u_{\mathrm{abs}} \leq$ 0.48. Via grid search, they found the optimal parameters for the work-rest cycle to be a contraction lasting $22 \mathrm{sec}$ at an intensity of $u_{\text {abs }}=0.26$ followed 

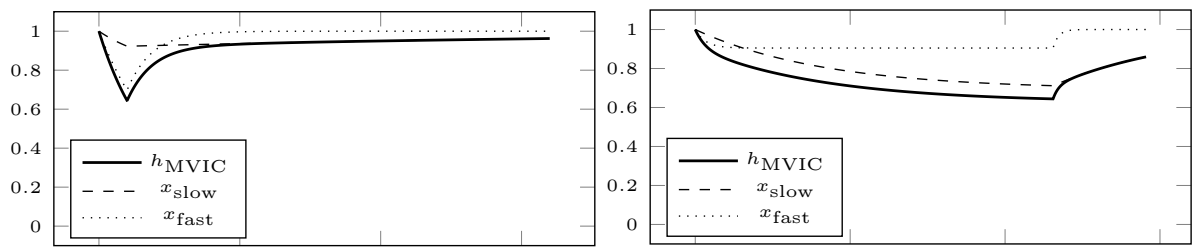

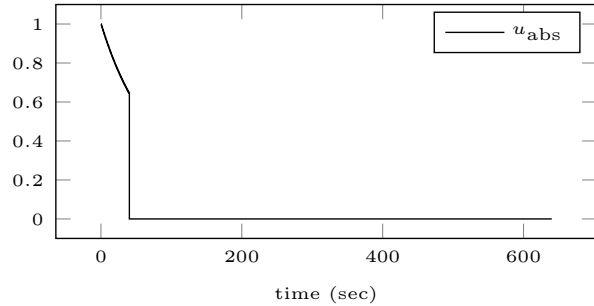

(a)

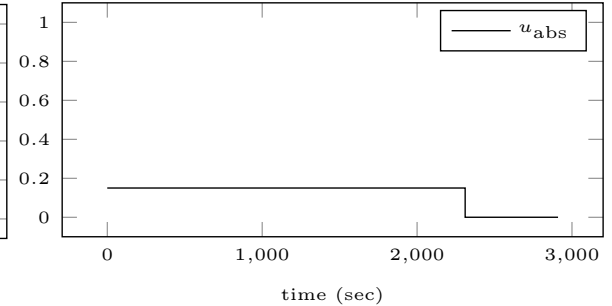

(b)

Figure 6: Model response obtained by simulating the calibrated model for an MVIC (a) and for a submaximal contraction (b) inducing the same level of fatigue followed by $10 \mathrm{~min}$ of recovery. The top row shows the model response and the absolute force input is illustrated in the bottom row. Recovery from the MVIC is almost complete after 10 min, whereas recovery from the submaximal contraction takes much longer.

by $38 \mathrm{sec}$ of rest. The left part of Figure 7 shows the model response obtained by simulating the calibrated model for this scenario. After $60 \mathrm{~min}$, MVIC force is reduced to $79.28 \%$.

We now compute a work-rest schedule that minimizes fatigue for the elbow flexors. We strive to reach the same FTI of 343.20 during $T=60$ min. Here, we allow changes of the temporal pattern and the force of the 60 work-rest cycles. Additionally, we add several safety requirements. First, MVIC force should not fall below $74.81 \%$ of baseline, which is the minimum value obtained when simulating the scenario motivated by Wood et al. 74]. Second, $u_{\text {abs }}$ should not exceed 0.48 , which is the upper limit used by Wood et al. 74]. Third, to obtain a real-life feasible work schedule, the maximum duration of a contraction should not exceed $30 \mathrm{sec}$ and at least $30 \mathrm{sec}$ should separate two contractions.

Remembering that $x_{\text {slow }} x_{\text {fast }}=h_{\mathrm{MVIC}}$ and setting $x=\left(x_{\text {slow }}, x_{\mathrm{fast}}, x_{\mathrm{FTI}}\right)^{T}$, the corresponding multi-stage optimal control problem [42] then reads as

$$
\begin{aligned}
\max _{x^{i}(\cdot), u_{\text {abs }}^{i}(\cdot), T^{i}} & x_{\text {slow }}^{119}\left(T^{119}\right) x_{\text {fast }}^{119}\left(T^{119}\right) \\
\text { s.t. } & \sum_{i=0}^{119} T^{i}=T \\
& x_{\mathrm{FTI}}^{119}\left(T^{119}\right)=343.20 \\
& x^{0}(0)=(1,1,0)^{T}
\end{aligned}
$$




$$
x^{i}(0)=x^{i-1}\left(T^{i-1}\right) \text { for } i \in\{1, \ldots, 119\}
$$

and for $i \in\{0,2, \ldots, 116,118\}$ and $t \in\left[0, T^{i}\right]$ :

$T^{i}<30 \mathrm{sec}$

$$
\frac{\mathrm{d}}{\mathrm{d} t} x_{\text {slow }}^{i}(t)=p_{1}\left(1-x_{\text {slow }}^{i}(t)\right)-p_{2} u_{\text {abs }}^{i}(t)
$$

$\frac{\mathrm{d}}{\mathrm{d} t} x_{\text {fast }}^{i}(t)=p_{3}\left(1-u_{\mathrm{abs}}^{i}(t)\right)^{p_{4}}\left(1-x_{\text {fast }}^{i}(t)\right)-p_{5} u_{\text {abs }}^{i}(t)$

$0.7481 \leq x_{\text {slow }}^{i}(t) x_{\text {fast }}^{i}(t)$

$0 \leq u_{\text {abs }}^{i}(t) \leq 0.48$

and for $i \in\{1,3, \ldots, 117,119\}$ and $t \in\left[0, T^{i}\right]$ :

$$
\begin{aligned}
& 30 \text { sec } \leq T^{i} \\
& \frac{\mathrm{d}}{\mathrm{d} t} x^{i}{ }_{\text {slow }}(t)=p_{1}\left(1-x_{\text {slow }}^{i}(t)\right) \\
& \frac{\mathrm{d}}{\mathrm{d} t} x^{i}{ }_{\text {fast }}(t)=p_{3}\left(1-x_{\text {fast }}^{i}(t)\right) \\
& 0.7481 \leq x_{\text {slow }}^{i}(t) x_{\text {fast }}^{i}(t)
\end{aligned}
$$

The superscript $i$ denotes the model stage index. During even stages, contractions with $u_{\text {abs }} \leq 0.48$ are possible. Odd stages are considered rest periods. The length $T^{i}$ of each stage is optimized.

To solve the problem, we again employ a first-discretize-then-optimize strategy. The optimal control software MUSCOD-II [42, 43, which originated from the work of Bock and Plitt [8, implements a direct multiple shooting approach and is used throughout the rest of this section.

The right part of Figure 7 shows the result of the optimization. Using high intensity contractions in the beginning allows to end the task earlier and yields an MVIC force of $90.19 \%$ of baseline after $60 \mathrm{~min}$. This is an improvement of $10.91 \%$ compared to the results obtained by adopting the scenario of Wood et al. 74] for the elbow flexors. To minimize fatigue at the end of the task, it is beneficial to accumulate work in the beginning. This can be done by either prolonging the contractions or increasing their intensity. Thus, during most of the task, at least one of the Constraints $(9 \mathrm{~g}),(9 \mathrm{j}),(9 \mathrm{k}),(9 \mathrm{~m})$, and $(9 \mathrm{p})$ is active. Note that, although the plots might suggest otherwise, all constraints are satisfied during the whole task.

Problem (9) contains initial conditions, endpoint constraints, control constraints, and state constraints and was chosen as a representative of a very general class of optimal control problems which can be solved with this approach. This emphasizes the advantages of an algorithmic design of loading schemes since it allows to treat even complex scenarios where intuitive planning would fail. 

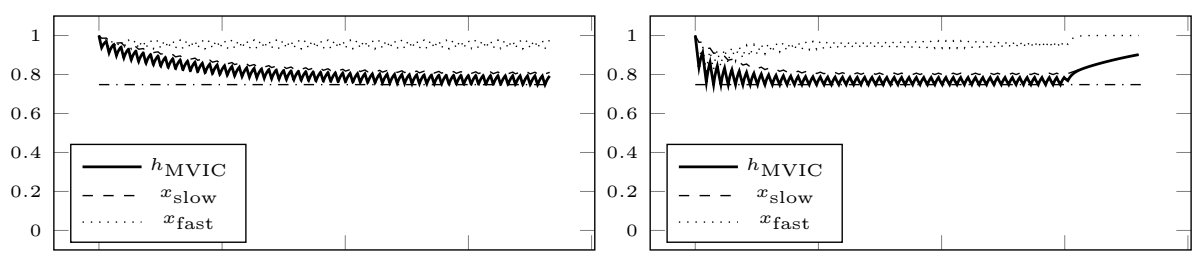

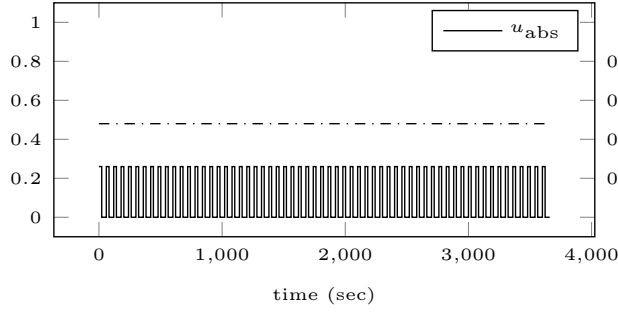

(a)

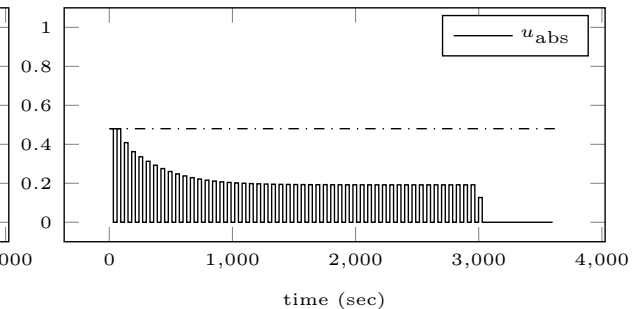

(b)

Figure 7: Model response obtained by simulating the calibrated model for the scenario adopted from Wood et al. [74] (a) and for an optimal loading scheme that minimizes fatigue with respect to certain safety requirements (b). The top row shows the model response and the absolute force input is illustrated in the bottom row. The horizontal dash-dotted line in the top row depicts the chosen constraint on MVIC force, whereas in the bottom row it depicts the maximum allowed external load. Using the optimal loading scheme, fatigue at the end of the task is less than when using the adopted scheme.

\subsection{Designing an optimal isometric RT session}

As a second example, we optimize an isometric RT session with the goal to increase MVIC strength of the elbow flexors. Major determinants of long-term adaptations include contraction duration, contraction intensity, inter-set rest, inter-repetition rest, and training volume [21. As the American College of Sports Medicine 2] recommends high loads for increasing maximal strength, we choose to maximize the FTI accumulated above a threshold intensity of $u_{\text {abs }}=0.8$, which we define as

$$
\mathrm{FTI}_{\geq 0.8} \stackrel{\text { def }}{=} \int_{0}^{T} \begin{cases}u_{\mathrm{abs}}(t) & \text { if } 0.8 \leq u_{\mathrm{abs}}(t) \\ 0 & \text { else } \mathrm{d} t .\end{cases}
$$

The left part of Figure 8 shows an intuitively planned isometric RT session consisting of 6 sets with 6 MVICs per set each lasting 4 sec, an inter-repetition rest of $18 \mathrm{sec}$, and an inter-set rest of $180 \mathrm{sec}$. This session is motivated by a similar one designed by Maffiuletti and Martin [49] for isometric RT of the knee extensors. However, their primary goal was not to increase strength but to examine the adaptations to different rates of force development. In contrast to the progressive increase of contraction intensity used by the authors, we choose 
to contract maximally during the $4 \mathrm{sec}$ of a repetition, as we want to maximize the $\mathrm{FTI}_{\geq 0.8}$. Following this plan, we can accumulate an $\mathrm{FTI}_{\geq 0.8}$ of 125.0.

We now formulate and solve an optimal control problem which maximizes $\mathrm{FTI}_{\geq 0.8}$. To allow a fair comparison to the adopted training plan, we limit the total time to $T=1584 \mathrm{sec}$ and the number of repetitions to 36 . Setting $x=\left(x_{\text {slow }}, x_{\text {fast }}\right)$, the corresponding multi-stage optimal control problem [42] then reads as

$$
\begin{aligned}
& \max _{x^{i}(\cdot), u_{\mathrm{abs}}^{i}(\cdot), T^{i}} \sum_{i \in\{0,2, \ldots, 68,70\}} \int_{0}^{T^{i}} u_{\mathrm{abs}}^{i}(t) \mathrm{d} t \\
& \text { s.t. } \sum_{i=0}^{70} T^{i} \leq T \\
& \quad x^{0}(0)=(1,1)^{T} \\
& \quad x^{i}(0)=x^{i-1}\left(T^{i-1}\right) \text { for } i \in\{1, \ldots, 70\} \\
& \text { and for } i \in\{0,2, \ldots, 68,70\} \text { and } t \in\left[0, T^{i}\right]: \\
& \quad \frac{\mathrm{d}}{\mathrm{d} t} x_{\text {slow }}^{i}(t)=p_{1}\left(1-x_{\text {slow }}^{i}(t)\right)-p_{2} u_{\text {abs }}^{i}(t) \\
& \quad \frac{\mathrm{d}}{\mathrm{d} t} x_{\text {fast }}^{i}(t)=p_{3}\left(1-u_{\text {abs }}^{i}(t)\right)^{p_{4}}\left(1-x_{\text {fast }}^{i}(t)\right)-p_{5} u_{\text {abs }}^{i}(t) \\
& \quad 0.8 \leq u_{\text {abs }}^{i}(t) \leq x_{\text {slow }}^{i}(t) x_{\text {fast }}^{i}(t)
\end{aligned}
$$

The superscript $i$ denotes the model stage index. During even stages, contractions with $0.8 \leq u_{\text {abs }}$ are possible. Odd stages are considered rest periods. The length $T^{i}$ of each stage is optimized.

The right part of Figure 8 depicts the result of the optimization. Instead of intermittent contractions grouped into sets, the solution consists of 36 single MVICs of decreasing duration and slightly varying inter-set rests. Here, we can accumulate an $\mathrm{FTI}_{\geq 0.8}$ of 172.0 . Thus, we could increase the $\mathrm{FTI}_{\geq 0.8}$ by 41 $\%$. Since all proposed contractions are maximal, the upper part of Constraint (11h) is active during all of them. The lower part of Constraint (11h is active at the end of each contraction. Our results would therefore support the design of isometric RT sessions without the grouping of repetitions into sets, if the goal is to maximize the FTI above a certain threshold. This was for example done by Carolan and Cafarelli [12, who employed 30 single MVICs each lasting 3 $4 \mathrm{sec}$ separated by more than $30 \mathrm{sec}$ to strengthen the knee extensors.

Naturally, the improvement and outcome depends highly on the chosen intuitive setting and objective functional. Problem (11) was chosen as a further representative of complex real-life problems which can be solved with this ap- 

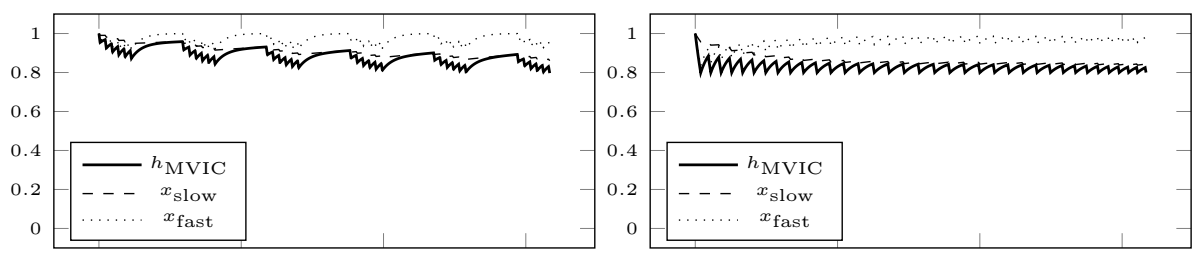

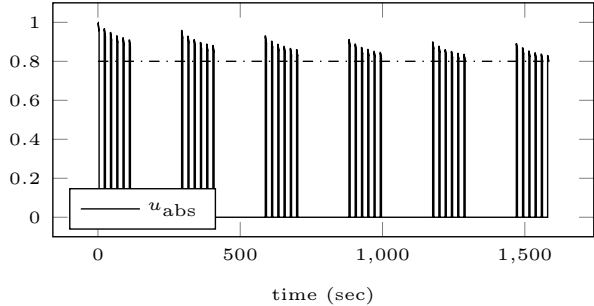

(a)

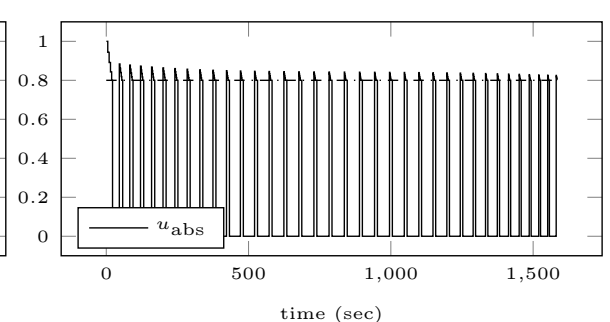

(b)

Figure 8: Model response obtained by simulating the calibrated model for the scenario motivated by Maffiuletti and Martin [49, (a) and for an optimal isometric RT session (b). The top row shows the model response and the absolute force input is illustrated in the bottom row. The horizontal dash-dotted line in the bottom row depicts the chosen intensity threshold. Using the optimal training plan results in a higher $\mathrm{FTI}_{\geq 0.8}$ than when using the intuitive plan.

proach. Depending on the individual goals, a broad variety of optimal control problems could be formulated.

\section{Conclusion}

Based on the model of Freund and Takala 22, we developed a simple and predictive ODE model of the time course of MVIC force which accounts for the fast and slow dynamics observed during fatiguing contractions and subsequent recovery [13. The model was validated with a comprehensive set of published data from the elbow flexors [25, 69, 70, 66, 65] and showed promising results. Once suitable data becomes available, it will be interesting to see how the model performs for submaximal intermittent contractions, for other muscle groups, and for individual subjects.

A special focus of this work was to keep the model suitable for derivativebased optimization methods. The model can readily be employed to optimize complex loading schemes, e.g. work shifts or resistance training plans. We demonstrated this by computing a work-rest schedule that minimizes fatigue and an optimal isometric RT session as examples.

To realize the full potential of the new model, suitable optimal control problems have to be developed and solved, e.g. to minimize the risk for work-related injuries or to maximize the benefits of resistance training. For the latter, current 
recommendations from the sports sciences about 'optimal' training with respect to maximizing strength, hypertrophy, power, or local muscular endurance have to be formulated mathematically, as we demonstrated exemplary in Section 6.3 . Furthermore, to facilitate fitting the model to individual persons and muscle groups, optimal experiments should be designed. For this purpose, available methods [39] can be used. Together with an extension to the more commonly used dynamic contractions, these tasks will be the topics of future research.

\section{Acknowledgments}

We gratefully thank Dr. Janet L. Taylor of Neuroscience Research Australia, Sydney, Australia for providing and explaining the experimental data of the studies Taylor et al. [69], Taylor et al. [70, Søgaard et al. [66], and Smith et al. 65.

\section{Grants}

JLH acknowledges support from the Heidelberg Graduate School of Mathematical and Computational Methods for the Sciences (DFG Graduate School 220), funded by the German Excellence Initiative.

\section{Disclosures}

No conflicts of interest, financial or otherwise, are declared by the authors.

\section{Author contributions}

JLH and CK conceived the idea for this work. JLH conducted the literature research, developed the model, performed the numerical experiments, and drafted the manuscript. JLH, CK, and JPS discussed and edited the draft. JLH, CK, and JPS approved the final version of the manuscript.

\section{Supplementary material}

A Python script for simulation of the proposed model is available alongside this article on bioRxiv.org.

\section{References}

[1] Allen DG, Lamb GD, Westerblad H. Skeletal Muscle Fatigue: Cellular Mechanisms. Physiological Reviews, 88(1):287-332, 2008. ISSN 0031-9333. doi: 10.1152/physrev.00015.2007. URL http://physrev.physiology. org/content/88/1/287.

[2] American College of Sports Medicine. American College of Sports Medicine position stand. Progression models in resistance training for healthy adults. Medicine and Science in Sports and Exercise, 41(3): 
687, 2009. URL http://journals.lww.com/acsm-msse/Fulltext/2009/ 03000/Progression_Models_in_Resistance_Training_for.26.aspx.

[3] Barnes WS. The relationship between maximum isometric strength and intramuscular circulatory occlusion. Ergonomics, 23(4):351-357, 1980. doi: 10.1080/00140138008924748. URL http://dx.doi.org/10.1080/ 00140138008924748. PMID: 7202390.

[4] Bauer I. Numerische Verfahren zur Lösung von Anfangswertaufgaben und zur Generierung von ersten und zweiten Ableitungen mit Anwendungen bei Optimierungsaufgaben in Chemie und Verfahrenstechnik. Dissertation, Heidelberg University, January 1999. URL http://doi.org/10.11588/ heidok.00001513.

[5] Bigland-Ritchie BR, Dawson NJ, Johansson RS, Lippold OC. Reflex origin for the slowing of motoneurone firing rates in fatigue of human voluntary contractions. The Journal of Physiology, 379(1):451-459, 1986. URL http://onlinelibrary.wiley.com/doi/10.1113/jphysiol.1986. sp016263/full.

[6] Bock HG. Numerical Treatment of Inverse Problems in Chemical Reaction Kinetics, pages 102-125. Springer Berlin Heidelberg, 1981. ISBN 978-3642-68220-9. URL http://dx .doi .org/10.1007/978-3-642-68220-9_8.

[7] Bock HG. Randwertproblemmethoden zur Parameteridentifizierung in Systemen nichtlinearer Differentialgleichungen. Bonner Mathematische Schriften, 183, 1987.

[8] Bock HG, Plitt KJ. A Multiple Shooting Algorithm for Direct Solution of Optimal Control Problems. In Proceedings of the 9th IFAC World Congress, page 242-247. Pergamon Press, Oxford, 1984.

[9] Brown LE, Weir JP. ASEP Procedures Recommendation I: Accurate Assessment Of Muscular Strength And Power. Professionalization of Exercise Physiology, 4(11), 2001. URL https://www.asep.org/asep/asep/ Brown2.pdf.

[10] Callahan DM, Umberger BR, Kent-Braun JA. A Computational Model of Torque Generation: Neural, Contractile, Metabolic and Musculoskeletal Components. PLOS ONE, 8(2):1-11, 02 2013. doi: 10.1371/journal.pone. 0056013. URL https://doi.org/10.1371/journal.pone.0056013.

[11] Callahan DM, Umberger BR, Kent JA. Mechanisms of in vivo muscle fatigue in humans: investigating age-related fatigue resistance with a computational model. The Journal of Physiology, 594(12):3407-3421, 2016. ISSN 1469-7793. doi: 10.1113/JP271400. URL http://dx.doi.org/10. 1113/JP271400. 
[12] Carolan B, Cafarelli E. Adaptations in coactivation after isometric resistance training. Journal of Applied Physiology, 73(3):911-917, 1992. ISSN 8750-7587. URL http://jap.physiology.org/content/73/3/911.

[13] Carroll TJ, Taylor JL, Gandevia SC. Recovery of central and peripheral neuromuscular fatigue after exercise. Journal of Applied Physiology, 122 (5):1068-1076, 2017. doi: 10.1152/japplphysiol.00775.2016. URL https : //doi.org/10.1152/japplphysiol.00775.2016. PMID: 27932676.

[14] Clarke DH. Strength Recovery from Static and Dynamic Muscular Fatigue. Research Quarterly. American Association for Health, Physical Education and Recreation, 33(3):349-355, 1962. doi: 10.1080/10671188. 1962.10616463. URL http://www.tandfonline.com/doi/abs/10.1080/ 10671188.1962.10616463.

[15] Contessa P, Luca CJD. Neural control of muscle force: indications from a simulation model. Journal of Neurophysiology, 109(6):1548-1570, 2013. ISSN 0022-3077. doi: 10.1152/jn.00237.2012. URL http://jn. physiology.org/content/109/6/1548.

[16] Deeb JM, Drury CG, Pendergast DR. An exponential model of isometric muscular fatigue as a function of age and muscle groups. Ergonomics, 35 (7-8):899-918, 1992. doi: 10.1080/00140139208967370. URL http://dx. doi.org/10.1080/00140139208967370. PMID: 1633796.

[17] Dideriksen JL, Farina D, Baekgaard M, Enoka RM. An integrative model of motor unit activity during sustained submaximal contractions. Journal of Applied Physiology, 108(6):1550-1562, 2010. ISSN 8750-7587. doi: 10.1152/japplphysiol.01017.2009. URL http://jap.physiology. org/content/108/6/1550.

[18] El ahrache K, Imbeau D, Farbos B. Percentile values for determining maximum endurance times for static muscular work. International Journal of Industrial Ergonomics, 36(2):99-108, 2006. ISSN 0169-8141. doi: http://dx.doi.org/10.1016/j.ergon.2005.08.003. URL http://www. sciencedirect.com/science/article/pii/S0169814105001344.

[19] Enoka RM, Duchateau J. Muscle fatigue: what, why and how it influences muscle function. The Journal of Physiology, 586(1):11-23, 2008. ISSN 1469-7793. doi: 10.1113/jphysiol.2007.139477. URL http://dx.doi.org/ 10.1113/jphysiol.2007.139477.

[20] Fayazi SA, Wan N, Lucich S, Vahidi A, Mocko G. Optimal pacing in a cycling time-trial considering cyclist's fatigue dynamics. In 2013 American Control Conference, pages 6442-6447, June 2013. doi: 10.1109/ACC.2013. 6580849. URL http://alirezafayazi.com/docs/06580849.pdf.

[21] Fleck SJ, Kraemer W. Designing Resistance Training Programs, 4E. Human Kinetics, 2014. 
[22] Freund J, Takala EP. A dynamic model of the forearm including fatigue. Journal of Biomechanics, 34(5):597-605, 2001. ISSN 0021-9290. doi: http://dx.doi.org/10.1016/S0021-9290(01)00009-4. URL http:// wWw.sciencedirect.com/science/article/pii/S0021929001000094.

[23] Fuglevand AJ, Winter DA, Patla AE. Models of recruitment and rate coding organization in motor-unit pools. Journal of Neurophysiology, 70 (6):2470-2488, 1993. ISSN 0022-3077. URL http://jn.physiology.org/ content/70/6/2470.

[24] Gandevia SC. Spinal and Supraspinal Factors in Human Muscle Fatigue. Physiological Reviews, 81(4):1725-1789, 2001. ISSN 0031-9333. URL http: //physrev.physiology.org/content/81/4/1725

[25] Gandevia SC, Allen GM, Butler JE, Taylor JL. Supraspinal factors in human muscle fatigue: evidence for suboptimal output from the motor cortex. The Journal of Physiology, 490(2):529-536, 1996. ISSN 1469-7793. doi: 10.1113/jphysiol.1996.sp021164. URL http://dx.doi.org/10.1113/ jphysiol.1996.sp021164.

[26] Gede G. Optimal Pacing Strategies for Cyclist Time Trials. PhD thesis, University of California, Davis, 2014. URL https://search.proquest. com/docview/1665571951.

[27] Gede G, Hubbard M. A bioenergetic model for simulating athletic performance of intermediate duration. Journal of Biomechanics, 47(14):3448 3453, 2014. ISSN 0021-9290. doi: http://dx.doi.org/10.1016/j.jbiomech. 2014.09.017. URL http://www.sciencedirect.com/science/article/ pii/S0021929014004837.

[28] Granata KP, Gottipati P. Fatigue influences the dynamic stability of the torso. Ergonomics, 51(8):1258-1271, 2008 . doi: 10.1080/00140130802030722. URL https://doi.org/10.1080/ 00140130802030722. PMID: 18608477.

[29] Grandjean E. Fatigue in industry. Occupational and Environmental Medicine, 36(3):175-186, 1979. ISSN 0007-1072. doi: 10.1136/oem.36. 3.175. URL http://oem. bmj . com/content/36/3/175.

[30] Hawkins D, Hull M. Muscle force as affected by fatigue: Mathematical model and experimental verification. Journal of Biomechanics, 26 (9):1117-1128, 1993. ISSN 0021-9290. doi: http://dx.doi.org/10.1016/ S0021-9290(05)80010-7. URL http://www. sciencedirect.com/science/ article/pii/S0021929005800107.

[31] Hawkins DA. A Cellular-based Muscle Model: Formulation and Application for Studying Muscle Mechanics. PhD thesis, University of California, Davis, 1990. AAI9102077. 
[32] Henneman E, Somjen G, Carpenter DO. Functional significance of cell size in spinal motoneurons. Journal of Neurophysiology, 28(3):560-580, 1965. ISSN 0022-3077. URL http://jn.physiology •org/content/28/3/560.

[33] Iguchi M, Baldwin K, Boeyink C, Engle C, Kehoe M, Ganju A, Messaros AJ, Shields RK. Low frequency fatigue in human quadriceps is fatigue dependent and not task dependent. Journal of Electromyography and Kinesiology, 18(2):308-316, 2008. ISSN 1050-6411. doi: http://dx. doi.org/10.1016/j.jelekin.2006.09.010. URL http://www. sciencedirect. com/science/article/pii/S1050641106001556.

[34] James A, Green S. A phenomenological model of muscle fatigue and the power-endurance relationship. Journal of Applied Physiology, 113(10): 1643-1651, 2012. ISSN 8750-7587. doi: 10.1152/japplphysiol.00800.2012. URL http://jap.physiology .org/content/113/10/1643.

[35] Kennedy DS, McNeil CJ, Gandevia SC, Taylor JL. Firing of antagonist small-diameter muscle afferents reduces voluntary activation and torque of elbow flexors. The Journal of Physiology, 591(14):3591-3604, 2013. ISSN 1469-7793. doi: 10.1113/jphysiol.2012.248559. URL http://dx.doi.org/ 10.1113/jphysiol.2012.248559.

[36] Keyserling MW, Herrin GD, Chaffin DB. Isometric strength testing as a means of controlling medical incidents on strenuous jobs. Journal of Occupational and Environmental Medicine, 22(5):332-336, 1980. URL https://www.ncbi.nlm.nih.gov/pubmed/7381613.

[37] Kircheis R. Structure Exploiting Parameter Estimation and Optimum Experimental Design Methods and Applications in Microbial Enhanced Oil Recovery. Dissertation, Heidelberg University, 2015. URL http://www. ub.uni-heidelberg.de/archiv/22098.

[38] Kisner C, Colby LA, Borstad J. Therapeutic exercise: foundations and techniques. F.A. Davis Company, Philadelphia, USA, 7th edition, 2017.

[39] Körkel S. Numerische Methoden für Optimale Versuchsplanungsprobleme bei nichtlinearen DAE-Modellen. Dissertation, Heidelberg University, 2002. URL http://www .ub.uni-heidelberg.de/archiv/2980.

[40] Law LAF, Avin KG. Endurance time is joint-specific: A modelling and meta-analysis investigation. Ergonomics, 53(1):109-129, 2010. doi: 10. 1080/00140130903389068. URL http://www.tandfonline.com/doi/abs/ 10.1080/00140130903389068. PMID: 20069487.

[41] Leetun DT, Ireland ML, Willson JD, Ballantyne BT, Davis IM. Core stability measures as risk factors for lower extremity injury in athletes. Medicine and Science in Sports and Exercise, 36(6):926-934, 2004. URL https://doi.org/10.1249/01.MSS.0000128145.75199.C3. 
[42] Leineweber DB, Bauer I, Bock HG, Schlöder JP. An efficient multiple shooting based reduced SQP strategy for large-scale dynamic process optimization. Part 1: theoretical aspects. Computers 83 Chemical Engineering, 27(2):157-166, 2003. ISSN 0098-1354. doi: https://doi.org/10.1016/ S0098-1354(02)00158-8. URL http://www.sciencedirect.com/science/ article/pii/S0098135402001588.

[43] Leineweber DB, Schäfer A, Bock HG, Schlöder JP. An efficient multiple shooting based reduced SQP strategy for large-scale dynamic process optimization. Part II: Software aspects and applications. Computers \&3 Chemical Engineering, 27(2):167-174, 2003. ISSN 0098-1354. doi: https://doi.org/10.1016/S0098-1354(02)00195-3. URL http://www . sciencedirect.com/science/article/pii/S0098135402001953.

[44] Liu JZ, Brown RW, Yue GH. A Dynamical Model of Muscle Activation, Fatigue, and Recovery. Biophysical Journal, 82(5):2344-2359, 2002. ISSN 0006-3495. doi: http://dx.doi.org/10.1016/S0006-3495(02) 75580-X. URL http://www.sciencedirect.com/science/article/pii/ S000634950275580X

[45] Looft JM. Adaptation and validation of an analytical localized muscle fatigue model for workplace tasks. PhD thesis, The University of Iowa, 2014. URL http://ir.uiowa.edu/etd/1482/

[46] Ma L, Chablat D, Bennis F, Zhang W. A new simple dynamic muscle fatigue model and its validation. International Journal of Industrial Ergonomics, 39(1):211-220, 2009. ISSN 0169-8141. doi: http://dx.doi. org/10.1016/j.ergon.2008.04.004. URL http://www.sciencedirect.com/ science/article/pii/S0169814108000863

[47] Ma L, Chablat D, Bennis F, Zhang W, Guillaume F. A new muscle fatigue and recovery model and its ergonomics application in human simulation. Virtual and Physical Prototyping, 5(3):123-137, 2010. doi: 10.1080/17452759.2010.504056. URL http://dx.doi.org/10.1080/ 17452759.2010 .504056 .

[48] Maffiuletti NA. Physiological and methodological considerations for the use of neuromuscular electrical stimulation. European Journal of Applied Physiology, 110(2):223-234, Sep 2010. ISSN 1439-6327. doi: 10.1007/s00421-010-1502-y. URL http://dx.doi.org/10.1007/ s00421-010-1502-y.

[49] Maffiuletti NA, Martin A. Progressive versus rapid rate of contraction during $7 \mathrm{wk}$ of isometric resistance training. Medicine and Science in Sports and Exercise, 33(7):1220-1227, July 2001. ISSN 0195-9131. doi: 10.1097/00005768-200107000-00022. URL http://dx.doi.org/10.1097/ 00005768-200107000-00022. 
[50] Mitchell M, Muftakhidinov B, Winchen T et al. Engauge Digitizer Software. URL http://markummitchell.github.io/engauge-digitizer.

[51] Monod H, Scherrer J. Capacité de travail statique d'un groupe musculaire synergique chez l'homme. Comptes Rendus des Séances de la Société de Biologie et de ses Filiales, 151(7):1358-1362, 1957.

[52] Monod H, Scherrer J. The work capacity of a synergic muscular group. Ergonomics, 8(3):329-338, 1965. doi: 10.1080/00140136508930810. URL http://dx.doi.org/10.1080/00140136508930810.

[53] Potvin JR, Fuglevand AJ. A motor unit-based model of muscle fatigue. PLOS Computational Biology, 13(6):1-30, 06 2017. doi: 10.1371/journal. pcbi.1005581. URL https://doi.org/10.1371/journal.pcbi.1005581.

[54] Rashedi E, Nussbaum MA. Quantifying the history dependency of muscle recovery from a fatiguing intermittent task. Journal of Biomechanics, 51: 26-31, 2017. ISSN 0021-9290. doi: http://dx.doi.org/10.1016/j.jbiomech. 2016.11.061. URL http://www.sciencedirect.com/science/article/ pii/S0021929016312404.

[55] Rich GQ. Muscular fatigue curves of boys and girls. Research Quarterly. American Association for Health, Physical Education and Recreation, 31 (3):485-498, 1960. doi: 10.1080/10671188.1960.10762056. URL https: //doi.org/10.1080/10671188.1960.10762056.

[56] Riener R, Quintern J, Schmidt G. Biomechanical model of the human knee evaluated by neuromuscular stimulation. Journal of Biomechanics, 29(9):1157-1167, 1996. ISSN 0021-9290. doi: 10.1016/0021-9290(96) 00012-7. URL http://www.sciencedirect.com/science/article/pii/ 0021929096000127 .

[57] Rodríguez-Rosell D, Pareja-Blanco F, Aagaard P, González-Badillo JJ. Physiological and methodological aspects of rate of force development assessment in human skeletal muscle. Clinical Physiology and Functional Imaging, pages n/a-n/a, 2017. ISSN 1475-097X. doi: 10.1111/cpf.12495. URL http://dx.doi.org/10.1111/cpf.12495.

[58] Rohmert W. Ermittlung von Erholungspausen für statische Arbeit des Menschen. Internationale Zeitschrift für angewandte Physiologie einschließlich Arbeitsphysiologie, 18(2):123-164, 1960. ISSN 1439-6327. doi: 10.1007/BF00698869. URL http://dx.doi.org/10.1007/BF00698869.

[59] Rozand V, Cattagni T, Theurel J, Martin A, Lepers R. Neuromuscular Fatigue Following Isometric Contractions with Similar Torque Time Integral. International Journal of Sports Medicine, 36(01):35-40, 2015. URL https://www.thieme-connect.de/DOI/DOI?10.1055/s-0034-1375614. 
[60] Sadamoto T, Bonde-Petersen F, Suzuki Y. Skeletal muscle tension, flow, pressure, and EMG during sustained isometric contractions in humans. European Journal of Applied Physiology and Occupational Physiology, 51 (3):395-408, Sep 1983. ISSN 1439-6327. doi: 10.1007/BF00429076. URL https://doi.org/10.1007/BF00429076.

[61] Sahlin K, Tonkonogi M, Söderlund K. Energy supply and muscle fatigue in humans. Acta Physiologica Scandinavica, 162(3):261266, 1998. URL http://onlinelibrary.wiley.com/doi/10.1046/j. 1365-201X.1998.0298f.x/full.

[62] Schlöder JP. Numerische Methoden zur Behandlung hochdimensionaler Aufgaben der Parameteridentifizierung. Dissertation. Rheinische FriedrichWilhelms-Universität Bonn, 1988.

[63] Shield A, Zhou S. Assessing Voluntary Muscle Activation with the Twitch Interpolation Technique. Sports Medicine, 34(4):253-267, 2004. ISSN 11792035. doi: 10.2165/00007256-200434040-00005. URL http://dx.doi.org/ 10.2165/00007256-200434040-00005.

[64] Sih B, Ng L, Stuhmiller J. Generalization of a Model Based on Biophysical Concepts of Muscle Activation, Fatigue and Recovery that Explains Exercise Performance. International Journal of Sports Medicine, 33(04):258-267, 2012. doi: 10.1055/ s-0031-1297958. URL https://www.thieme-connect.com/products/ ejournals/html/10.1055/s-0031-1297958.

[65] Smith JL, Martin PG, Gandevia SC, Taylor JL. Sustained contraction at very low forces produces prominent supraspinal fatigue in human elbow flexor muscles. Journal of Applied Physiology, 103(2):560568, 2007. ISSN 8750-7587. doi: 10.1152/japplphysiol.00220.2007. URL http://jap.physiology.org/content/103/2/560.

[66] Søgaard K, Gandevia SC, Todd G, Petersen NT, Taylor JL. The effect of sustained low-intensity contractions on supraspinal fatigue in human elbow flexor muscles. The Journal of Physiology, 573(2):511-523, 2006. ISSN 1469-7793. doi: 10.1113/jphysiol.2005.103598. URL http://dx.doi.org/ 10.1113/jphysiol.2005.103598.

[67] Sonne MW, Potvin JR. A modified version of the three-compartment model to predict fatigue during submaximal tasks with complex force-time histories. Ergonomics, 59(1):85-98, 2016. doi: 10.1080/00140139.2015. 1051597. URL http://dx.doi.org/10.1080/00140139.2015.1051597. PMID: 26018327.

[68] Tan B. Manipulating Resistance Training Program Variables to Optimize Maximum Strength in Men: A Review. The Journal of Strength \& Conditioning Research, 13(3):289-304, 1999. URL https: //insights.ovid.com/strength-conditioning-research/jscr/1999/ 
08/000/manipulating-resistance-training-program-variables/19/ 00124278 .

[69] Taylor JL, Butler JE, Gandevia SC. Altered responses of human elbow flexors to peripheral-nerve and cortical stimulation during a sustained maximal voluntary contraction. Experimental Brain Research, 127 (1):108-115, 1999. ISSN 1432-1106. doi: 10.1007/s002210050779. URL http://dx.doi.org/10.1007/s002210050779.

[70] Taylor JL, Allen GM, Butler JE, Gandevia SC. Supraspinal fatigue during intermittent maximal voluntary contractions of the human elbow flexors. Journal of Applied Physiology, 89(1):305-313, 2000. ISSN 8750-7587. URL http://jap.physiology.org/content/89/1/305

[71] Taylor JL, Amann M, Duchateau J, Meeusen R, Rice CL. Neural Contributions to Muscle Fatigue: From the Brain to the Muscle and Back Again. Medicine and Science in Sports and Exercise, 48(11):2294-306, 2016. URL https://www.ncbi.nlm.nih.gov/pubmed/27003703

[72] Todd G, Taylor JL, Gandevia SC. Measurement of voluntary activation of fresh and fatigued human muscles using transcranial magnetic stimulation. The Journal of Physiology, 551(2):661-671, 2003. ISSN 1469-7793. doi: 10.1113/jphysiol.2003.044099. URL http://dx.doi.org/10.1113/ jphysiol.2003.044099.

[73] Vøllestad NK. Measurement of human muscle fatigue. Journal of Neuroscience Methods, 74(2):219-227, 1997. ISSN 0165-0270. doi: 10.1016/ S0165-0270(97)02251-6. URL http://www.sciencedirect.com/science/ article/pii/S0165027097022516.

[74] Wood DD, Fisher DL, Andres RO. Minimizing Fatigue during Repetitive Jobs: Optimal Work-Rest Schedules. Human Factors, 39(1):83-101, 1997. doi: 10.1518/001872097778940678. URL http://dx.doi.org/10.1518/ 001872097778940678 . PMID: 9302881.

[75] Xia T, Law LAF. A theoretical approach for modeling peripheral muscle fatigue and recovery. Journal of Biomechanics, 41(14):3046-3052, 2008. ISSN 0021-9290. doi: http://dx.doi.org/10.1016/j.jbiomech.2008. 07.013. URL http://www.sciencedirect.com/science/article/pii/ S0021929008003692 